\title{
Targeting angiogenesis in small cell lung cancer
}

\author{
Michalis Stratigos ${ }^{1}$, Alexios Matikas ${ }^{1,2}$, Alexandra Voutsina ${ }^{3}$, Dimitrios Mavroudis ${ }^{1,3}$, Vassilis Georgoulias ${ }^{2,3}$ \\ ${ }^{1}$ Department of Medical Oncology, University General Hospital of Heraklion, Heraklion, Crete, Greece; ${ }^{2}$ Hellenic Oncology Research Group \\ (HORG), Athens, Greece; ${ }^{3}$ Laboratory of Translational Oncology, University of Crete, School of Medicine, Heraklion, Crete, Greece \\ Contributions: (I) Conception and design: A Voutsina, M Stratigos; (II) Administrative support: V Georgoulias, D Mavroudis; (III) Provision of study \\ materials or patients: None; (IV) Collection and assembly of data: M Stratigos, A Matikas; (V) Data analysis and interpretation: All authors; (VI) \\ Manuscript writing: All authors; (VII) Final approval of manuscript: All authors. \\ Correspondence to: Michalis Stratigos, MD. Department of Medical Oncology, University General Hospital of Heraklion, P.O. Box 1352, Heraklion, \\ 71110 Crete, Greece. Email: mstratig@gmail.com.
}

\begin{abstract}
Small cell lung cancer (SCLC) is a highly aggressive and lethal malignancy. Despite high initial response rates to systemic chemotherapy, the disease eventually relapses; further treatment only modestly improves outcomes and overall survival (OS) for patients with extensive stage disease is less than one year. Little progress has been made during the past decades, with no new drugs approved. Consequently, the development of novel strategies is an unmet need. The inhibition of angiogenesis, a defining characteristic of cancer, has demonstrated modest efficacy in several human malignancies, including non-small cell lung cancer (NSCLC). However, results from clinical trials in SCLC have been disappointing, and no anti-angiogenic agent has received regulatory approval due to lack of clinical efficacy. The elucidation of underlying mechanisms responsible for tumor resistance to angiogenic therapy and the simultaneous blockade of multiple elements that play a role in angiogenesis need to be further explored.
\end{abstract}

Keywords: Angiogenesis; bevacizumab (Bev); small cell lung cancer (SCLC); vascular endothelial growth factor (VEGF)

Submitted Jun 07, 2016. Accepted for publication Jul 07, 2016.

doi: 10.21037/tlcr.2016.08.04

View this article at: http://dx.doi.org/10.21037/tlcr.2016.08.04

\section{Introduction}

Small cell lung cancer (SCLC) represents approximately $15 \%$ of newly diagnosed lung cancer cases and it is closely correlated with smoking, since it very rarely appears in non-smokers and its incidence has been declining during the past decades, following decreasing smoking rates in Western countries (1). Its clinicopathological hallmarks are a short doubling time, high growth fraction and exquisite initial sensitivity to chemotherapy and radiotherapy. Almost $70 \%$ of patients have clinically overt metastatic disease at diagnosis (termed extensive disease, ED-SCLC); the remaining $30 \%$ has disease confined to one hemithorax which can be encompassed in a single radiotherapy field (limited disease, LD-SCLC). However, these patients are also presumed to harbor micrometastases, as evidenced by the poor survival rates from local treatment alone without systemic chemotherapy (2). Despite the striking initial sensitivity of SCLC to chemotherapy resulting in dramatic responses, acquired resistance and disease progression are commonly observed after a short period of time. Median overall survival (OS) with contemporary treatment is approximately 17 months for LD-SCLC and 9-11 months in ED-SCLC; anecdotal cases with ED-SCLC survive for 5 years (3).

Combination chemotherapy with etoposide and a platinum salt, either cisplatin or carboplatin, represent the gold standard chemotherapy regimen in SCLC for almost 30 years. Attempts to improve clinical outcomes by using other agents such as irinotecan (4), alternating regimens (5), increasing chemotherapy dose intensity (6) or dose density $(7,8)$, treatment duration (9) or adding a third (10) or fourth drug (11) have resulted in marginal, if any, improvements in OS. In pretreated patients, responses from chemotherapy 
occur less frequently and are of shorter duration and no agent or combination has demonstrated a prolongation of OS compared to the standard of care, topotecan $(12,13)$, with the exception of amrubicin as demonstrated in a subset analysis of refractory patients treated in the context of a randomized trial comparing amrubicin vs. topotecan (14). Therefore, more effective treatments are highly needed.

The inhibition of angiogenesis is generally an attractive therapeutic strategy in oncology. Its ubiquitous role in the pathogenesis of malignant progression is underscored by the clinically relevant improvements in progression-free survival (PFS) and overall response rate (ORR) (and, less commonly, in OS) reported in phase III trials of several human malignancies and despite toxicity and cost-related concerns, anti-angiogenic agents have become an essential part of the therapeutic armamentarium for many solid tumors. Unfortunately, the success in SCLC has been less prominent. Herein, we review relevant preclinical and clinical data regarding the role of angiogenesis and its inhibition in SCLC.

\section{Angiogenesis: a hallmark of cancer}

A large body of literature supports the significance of angiogenesis in tumor progression from its early stages to overt metastatic disease and therefore it has been recognized as a hallmark, a defining characteristic of cancer at the conceptual framework introduced by Weinberg and Hanahan (15). The processes of vasculogenesis (the formation of tubes by endothelial cells) and angiogenesis (the development of new blood vessels from pre-existing ones) are initiated early in the course of tumorigenesis and remain activated throughout the disease trajectory. The tumor neovasculature is largely abnormal, with distorted, leaking blood vessels and dysregulated endothelial cell function (16).

Angiogenesis is regulated by an angiogenic switch, a balance between pro-angiogenic factors such as the vascular endothelial growth factor A (VEGF-A) and antiangiogenic ones such as thrombospondin-1 (TSP-1) (17). The expression of these physiologic regulators is affected directly or indirectly by the presence of the malignancy, thus tipping the angiogenic switch to its activation. Hypoxia in the growing tumor stimulates angiogenesis by upregulating hypoxia inducible factors (HIF)-1a and HIF2a which in turn induce the production of VEGFs that bind to and activate VEGF receptors (VEGFR), thus activating the VEGF pathway which is central in the pathophysiology of angiogenesis (18). When VEGF binds to VEGFR2 on endothelial cells, the expression of the Notch ligand Delta-like 4 (DLL4) is increased. DLL4 then binds to its receptor Notch on the adjacent endothelium, and acts as a negative feedback regulator (19). Additional proangiogenic molecules, such as fibroblast growth factor (FGF) and angiopoietin-2 (Ang-2) also sustain the continuous activation of angiogenesis.

Bone marrow derived cells have also been shown to play important role in cancer-promoted angiogenesis. As an example, tumor-associated macrophages (TAMs) accumulate in hypoxic areas and stimulate angiogenesis, enhance tumor cell migration and invasion through the direct production of VEGF or its indirect release from the extracellular matrix via the production of matrix metalloproteinase 9 (MMP9) (20). Other mechanisms that involve marrow-derived cells include their integration to the tumor neovasculature and the protection of endothelial cells from the effects of treatment.

Inhibiting angiogenesis may be achieved using several different methods which include the binding of VEGF-A by monoclonal antibodies [bevacizumab (Bev)] or decoy receptors (aflibercept), the blockade of VEGFR2 using monoclonal antibodies (ramucirumab) and the use of oral tyrosine kinase inhibitors (TKIs) that bind to VEGFR domains. However, suppressing angiogenesis has been shown to induce adaptive responses by the tumor, with heightened invasion and metastasis capabilities which in return offer access to the tumor in the normal vasculature (21). This adaptive response implies that angiogenesis is a product of multiple, overlapping pathways and mechanisms and that its inhibition should be combined with other antineoplastic strategies in order to delay tumor progression, since blocking single components of the VEGF pathway leads to suboptimal results.

\section{Angiogenesis in SCLC}

Several lines of evidence suggest that angiogenesis in SCLC has a fundamental role in determining the growth rate, invasiveness and development of metastases; moreover, the formation of structurally and functionally abnormal neovessels from the existing blood vessels is considered to mediate resistance to chemotherapy $(22,23)$. Microvessel density is correlated with VEGF levels in SCLC and an increased microvessel count was an adverse prognostic factor in a study of patients with SCLC that were treated with surgery and adjuvant chemotherapy $(24,25)$.

Several components of the VEGF pathway have been shown to be overexpressed in SCLC and this overexpression 
has been linked to poor outcomes. Wan et al. demonstrated that HIF-1a promotes growth and angiogenesis in SCLC by upregulating the expression of angiogenic genes and proposed HIF-1a as a potential target in the treatment of SCLC (26). Interestingly, the blockade of c-kit inducible HIF-1a by imatinib has been shown to downregulate VEGF expression in SCLC cell lines (27). Unfortunately, imatinib failed to demonstrate efficacy in a clinical trial, even in patients selected for c-kit positivity (28).

VEGF levels are increased in SCLC patients compared to healthy controls $(29,30)$. Salven et al. were among the first to demonstrate that increased serum VEGF levels were the only independent prognostic factor except for tumor stage in untreated patients (31). Confirming these results, in the previously mentioned study by Lucchi et al., VEGF levels were an independent adverse prognostic factor in the adjuvant setting (25). Additionally, Zhan et al. performed a systematic review and meta-analysis of VEGF expression in lung cancer and again confirmed that VEGF is a prognostic factor in SCLC (32). Moreover, it has been demonstrated that SCLC cell lines express VEGFR2/3 receptors suggesting that the VEGF/VEGFR pathway is an autocrine growth regulator in SCLC (33). Taken together, these data underscore the fact that the VEGF pathway is activated in SCLC and this activation has important prognostic implications.

Other key mediators of angiogenesis have also been found to be overexpressed in SCLC. For example, two studies indicate that Ang-2 levels are elevated in SCLC compared to healthy controls and this increases an independent predictor for shorter OS $(34,35)$. Trebananib, an angiopoietin inhibitor, has been combined with the VEGF inhibitors Bev or motesanib in a phase $1 \mathrm{~b}$ trial in various solid tumors; only 2 enrolled patients had SCLC and none achieved a partial response to treatment (36). On the other hand, several studies and a recent systematic review and meta-analysis support both the predictive and prognostic capacity of basic FGF (bFGF) overexpression in SCLC (37-39). FGF receptor 1 (FGFR1) gene amplification and overexpression has also been documented in SCLC, offering a rationale for therapeutic targeting this pathway $(40,41)$. Although the FGFR inhibitor PD173074 was shown to block SCLC growth in vitro and in xenografts, its mechanism of action was postulated to stem from the induction of apoptosis rather than the inhibition of angiogenesis (42).

These observations illustrate the significance of angiogenesis in SCLC. Efforts to translate them from bench to bedside have been undertaken in the form of multiple, albeit relatively small, clinical trials which are summarized in Table 1.

\section{Targeting angiogenesis in SCLC}

$B e v$

$\mathrm{Bev}$ is a recombinant, humanized monoclonal antibody that binds to VEGF-A, thus inhibiting angiogenesis. It has been approved for use in several malignancies, and its efficacy has also been investigated in SCLC.

Ready et al. conducted a phase II trial (CALGB 30306) to evaluate the efficacy of adding Bev to cisplatin/irinotecan regimen (43). In this trial, 64 eligible patients with EDSCLC were treated with cisplatin and irinotecan plus Bev $15 \mathrm{mg} / \mathrm{kg}$ on day 1 of a 21-day cycle, for a maximum of 6 cycles. ORR was $75 \%$, the median PFS was 7.0 months and the median OS was 11.6 months. However, the 12-month survival rate was $43.8 \%$ which was lower than the prespecified threshold of $57 \%$. Interestingly lower pretreatment VEGF levels were associated with worse PFS, which is in contrast with results from previous studies, whereas patients developing hypertension had improved outcomes.

Similarly, in the randomized SALUTE trial (44), 102 patients were randomly assigned to chemotherapy with a platinum salt and etoposide plus Bev $15 \mathrm{mg} / \mathrm{kg}$ or placebo. After the completion of 4 cycles of chemotherapy, patients received maintenance Bev or placebo until disease progression. Median PFS was 4.4 months for the placebo $v s$. 5.5 months for the Bev group [hazard ratio (HR) 0.53; 95\% confidence interval (CI), 0.32-0.86]. However, median OS was 10.9 months for the placebo vs. 9.4 months for the Bev group (HR 1.16; 95\% CI, 0.66-2.04). Febrile neutropenia, diarrhea, gastrointestinal perforation, pneumonia and hypertension were more frequent in the Bev arm.

The E3501 study was a single arm phase II trial which enrolled 65 untreated patients with ED-SCLC (45). Patients were treated with cisplatin, etoposide and Bev for 4 cycles. Non-progressing patients continued on the same Bev schedule for a total of one year. The trial's primary endpoint was the 6-month PFS. After a median follow up of 27.5 months, median PFS was 4.7 months and the 6-month PFS was $30.2 \%$. Median OS was 10.9 months and the ORR was $63.5 \%$. Hematologic toxicity was common and febrile neutropenia occurred in $7.8 \%$ of patients. Baseline VEGF levels did not correlate with response to chemotherapy, however patients with high ICAM levels had a non- 
Table 1 Published trials of anti-angiogenic treatment in SCLC

\begin{tabular}{|c|c|c|c|c|c|c|c|}
\hline Agent & Reference & Phase & $\mathrm{N}$ & Trial design & ORR (\%) & PFS (months) & OS (months) \\
\hline \multirow[t]{8}{*}{ Bevacizumab } & Ready et al. (43) & II & 64 & CDDP/CPT-11/Bev & 75 & 7 & 11.6 \\
\hline & Spigel et al. (44) & $\|$ & 102 & $\mathrm{EP} \pm \mathrm{Bev}$ & $\begin{array}{l}58 \text { vs. } 48 \\
(P=0.3269)\end{array}$ & $\begin{array}{l}5.5 \text { vs. } 4.4 \\
(\mathrm{HR}=0.53)\end{array}$ & $\begin{array}{l}9.4 \text { vs. } 10.9 \\
(\mathrm{HR}=1.16)\end{array}$ \\
\hline & Horn et al. (45) & II & 65 & EP/Bev & 63.5 & 4.7 & 10.9 \\
\hline & Spigel et al. (46) & II & 51 & Carbo/CPT-11/Bev & 84 & 9.1 (TTP) & 12.1 \\
\hline & Petrioli et al. (48) & II & 22 & $\mathrm{EP} / \mathrm{Bev}$ & 77.2 & 7.8 & 13.2 \\
\hline & Jalal et al. (49) & II & 34 & Paclitaxel/Bev (pretreated) & 18.1 & 3.38 & 6.9 \\
\hline & Mountzios et al. (50) & II & - & Paclitaxel/Bev (pretreated) & 20 & 2.7 & 6.3 \\
\hline & Spigel et al. (51) & II & 50 & Topotecan/Bev (pretreated) & 16 & $\begin{array}{l}6.24 \text { (sensitive), } \\
2.91 \text { (refractory) }\end{array}$ & 7.4 \\
\hline \multirow{2}{*}{ Sunitinib } & Ready et al. (54) & II & 138 & $\mathrm{EP} \rightarrow$ sunitinib vs. placebo & - & $\begin{array}{l}3.7 \text { vs. } 2.1 \\
(P=0.02)\end{array}$ & $\begin{array}{l}9 \text { vs. } 6.9 \\
(P=0.16)\end{array}$ \\
\hline & Han et al. (55) & II & 25 & Second line, monotherapy & 9 & 1.4 & 5.6 \\
\hline \multirow[t]{2}{*}{ Thalidomide } & Pujol et al. (56) & III & 119 & $\mathrm{PCDE} \pm$ thalidomide & $\begin{array}{l}87 \text { vs. } 84 \\
(P=0.69)\end{array}$ & $\begin{array}{l}6.6 \text { vs. } 6.4 \\
(P=0.15)\end{array}$ & $\begin{array}{l}11.7 \text { vs. } 8.7 \\
(P=0.16)\end{array}$ \\
\hline & Lee et al. (57) & III & 724 & $\mathrm{CE} \pm$ thalidomide & 74 vs. 72 , (NS) & $\begin{array}{l}7.6 \text { vs. } 7.6 \\
(P=0.39)\end{array}$ & $\begin{array}{l}10.1 \text { vs. } 10.5 \\
(P=0.28)\end{array}$ \\
\hline Aflibercept & Allen et al. (58) & II & 192 & $\begin{array}{l}\text { Topotecan } \pm \text { aflibercept } \\
\text { (pretreated) }\end{array}$ & $\begin{array}{l}2 \text { vs. } 0 \\
(P=0.50)\end{array}$ & $\begin{array}{l}1.6 \text { vs. } 1.3 \\
(P=0.02)\end{array}$ & $\begin{array}{l}5.4 \text { vs. } 4.4 \\
(P=0.34)\end{array}$ \\
\hline Nintedanib & Han et al. (63) & II & 22 & Second, third line & 5 & 1.0 & 9.8 \\
\hline Rovalpituzumab & Pietanza et al. (64) & I & 73 & Second line & 24 & - & - \\
\hline
\end{tabular}

SCLC, small cell lung cancer; CDDP, cisplatin; CPT-11, irinotecan; Bev, bevacizumab; EP, etoposide/cisplatin; PCDE, cisplatin, cyclophosphamide, epidoxorubicin, etoposide; CE: carboplatin/etoposide; ORR, overall response rate; DCR, disease control rate; NS, statistically non-significant; NR, not reported; PFS, progression-free survival; TTP, time to progression; OS, overall survival; HR, hazard ratio; sensitive, platinum sensitive relapse; refractory, platinum refractory relapse.

significant trend towards improved OS (HR 0.48, $\mathrm{P}=0.06$ ).

In a phase II trial by Spigel et al. (46), 51 patients were treated with carboplatin plus irinotecan given in 28-day cycles, plus Bev at a $10 \mathrm{mg} / \mathrm{kg}$ dose given every 15 days, which was continued as maintenance treatment. Sixmonth time to progression (TTP) was $76 \%$ and ORR $84 \%$.
Median TTP was 9.1 months and median OS 12.1 months. In the recently published phase II-III IFCT-0802 trial by Pujol et al. (47), 147 patients received 2 cycles of chemotherapy with etoposide/cisplatin (EP) or PCDE (cisplatin/cyclophosphamide/epidoxorubicin/etoposide). A total of 103 patients $(70.1 \%)$ with a partial response were 
randomized to continue with chemotherapy alone for a maximum of 6 cycles or chemotherapy plus Bev $7.5 \mathrm{mg} / \mathrm{kg}$ on day 1 every 21 days, until a maximum of 2 years. Disease control rate (DCR) at the end of the fourth chemotherapy cycle was used as a phase II endpoint, and was similar between groups $(89.2 \%$ in the chemotherapy alone $v s$. $91.9 \%$ in the combination group, $\mathrm{P}=1.0$ ). After a median follow up of 37.7 months, PFS did not differ significantly between groups (5.5 vs. 5.3 months, $\mathrm{P}=0.82$ ). Median OS was also similar between groups (13.3 vs. 11.1 months). In a biomarker analysis, neither serum VEGF nor soluble VEGF receptor titrations were either prognostic or predictive of Bev's effect on outcome.

Recently, a study by Petrioli et al. evaluated 22 patients with chemonaive SCLC, who were treated with 3 cycles of cisplatin/etoposide followed by 3 cycles of cisplatin/ etoposide plus Bev $15 \mathrm{mg} / \mathrm{kg}$ for non-progressing patients. After completion of a total of six cycles of treatment, nonprogressing patients received oral etoposide plus Bev until disease progression. ORR was $77.2 \%$ and 9 -month DCR was $36.3 \%$. Median PFS was 7.8 months and median OS was 13.2 months (48).

Jalal et al. also reported a phase II trial in 34 pre-treated patients with chemosensitive relapsed SCLC treated with weekly paclitaxel plus biweekly Bev, with the option of maintenance Bev until disease progression. The median PFS was 14.7 weeks, ORR was $18.1 \%$ and median OS was 30 weeks. Although the combination was relatively safe, it did not provide benefit compared with historical controls and the authors concluded that no further investigation is warranted (49).

In another trial by the Hellenic Oncology Research Group in patients with chemoresistant SCLC, the combination of paclitaxel with Bev produced a 20\% ORR, median PFS of 2.7 months and median OS of 6.3 months (50).

In an attempt to improve outcomes offered by topotecan as second line treatment of SCLC, Spigel $e t$ al. published the results of a phase II single arm trial of 50 patients with relapsed SCLC who were treated with oral topotecan $2.3 \mathrm{mg} / \mathrm{m}^{2}$ on days $1-5$ plus Bev $15 \mathrm{mg} / \mathrm{kg}$ given every 21 days. The primary endpoint of the study, 3-month PFS, was $65 \%$ which was promising compared with the historical control of $50 \%(\mathrm{P}=0.017)$ but did not meet the predefined criteria for clinically meaningful improvement (51).

\section{Sunitinib}

Sunitinib is a TKI which inhibits VEGF receptors, platelet-derived growth factor receptor (PDGFR), Flt3 and c-Kit. It is approved for use in renal cell carcinoma, gastrointestinal stromal tumors (GISTs) and advanced pancreatic neuroendocrine tumors. Sunitinib has been studied as maintenance treatment and as second line treatment in patients with SCLC, with mostly negative results.

In a phase II trial by Schneider et al., sixteen patients diagnosed with ED-SCLC who had not progressed after a maximum of four cycles of treatment with a platinum and etoposide regimen, were treated with sunitinib $50 \mathrm{mg}$ orally once daily for four consecutive weeks followed by a twoweek rest period (6-week cycle). Treatment was continued until disease progression or unacceptable toxicity. The trial's primary endpoint was 4-month PFS and median treatment duration was 4 weeks. Although no objective responses were observed, four patients (25\%) achieved disease stabilization. Median PFS was 2.5 months and the 4-month PFS rate was $13 \%$. The most commonly reported grade $3 / 4$ toxicities were thrombocytopenia (25\%), fatigue (19\%), muscle weakness (13\%) and hypothyroidism (6\%) (52). A possible drawback of this study was the $4 / 2$ week sunitinib administration schedule, which could be considered suboptimal, as studies in renal cell carcinoma have shown that during the 2-week rest period, both VEGFRs levels and FDG avidity increase $(65,66)$.

In another phase II maintenance trial by Spigel et al. (53), 34 eligible patients with extensive stage SCLC were treated with carboplatin plus weekly irinotecan. Patients who achieved disease control were treated with sunitinib $25 \mathrm{mg}$ daily until disease progression or unacceptable toxicity. The trial's primary endpoint was 1-year survival rate. After a median follow up of 50 weeks, and a median duration of treatment of 9 weeks, partial response was noted in $56 \%$ of patients and complete response in 3\%. One-year survival rate was $54 \%$.

More recently, the results of a larger randomized phase II trial by Cancer and Leukemia Group B (GALGB 30504) were published (54). In this trial, 138 patients with EDSCLC received 4-6 cycles of induction chemotherapy, consisting of a platinum salt plus etoposide. Ninety-five patients whose disease had not progressed were randomized to receive maintenance sunitinib $(150 \mathrm{mg}$ loading dose, then $37.5 \mathrm{mg}$ daily) or placebo until disease progression. Patients with objective response were offered prophylactic cranial irradiation (PCI), whereas patients progressing on placebo could cross over to sunitinib. The trial's primary endpoint was PFS. Median PFS was 2.1 months for placebo vs. 3.7 months 
for sunitinib (HR 1.62; 95\% CI, 1.02-2.60; $\mathrm{P}=0.02$ ), whereas median OS was 6.9 months for placebo vs. 9.0 months for sunitinib (HR 1.28; 95\% CI, 0.79-2.10; $\mathrm{P}=0.16$ ). Significantly more patients in the sunitinib arm experienced grade $3 / 4$ adverse events compared with placebo, namely fatigue (19\%), neutropenia (14\%), leukopenia (7\%) and thrombocytopenia (7\%).

Equally disappointing were the results of a Korean phase II study (55), which enrolled 25 patients with extensivestage SCLC who had progressed after a platinum-based regimen (62\% refractory and 38\% sensitive). Patients were treated with sunitinib $50 \mathrm{mg}$ in a $4 / 2$ week fashion, for a median of 6.8 weeks and were followed for a median of 6 months. ORR was 9\%, DCR was 39\%, median PFS was 1.4 months and median OS 5.6 months. The trial failed to meet its primary endpoint of a $35 \%$ ORR.

In summary, substantial toxicity caused by exposure to sunitinib in combination with marginal, clinically nonmeaningful benefits in ORR and PFS mean that further evaluation of this agent in SCLC is not warranted.

\section{Thalidomide}

Thalidomide, although infamous for its teratogenic potential, has been in clinical use for several decades. It has been approved for use in multiple myeloma and has also been evaluated in SCLC and other malignancies. Its antineoplastic action is thought to be a result of its anti-angiogenic and immunomodulatory properties. Thalidomide appears to inhibit inflammation through suppression of NF-kB, COX2, TNF, IL-1 and IL-6 and stimulates T-cell activity and Th-1 cell differentiation. Although its immunomodulatory mechanism of action is well documented, its antiangiogenic role in SCLC is disputed, as it is believed that thalidomide's anti-angiogenic effect could be a result of its anti-inflammatory action. SCLC is poorly infiltrated with inflammatory cells, so angiogenesis could be an irrelevant target. Moreover, thalidomide is thought to restore endothelial cell protease-activate receptor 1 expression (67), which in turn induces proliferation of alveolar capillary endothelial cells, thus stimulating angiogenesis. Decrease in tumor vascular permeability and interstitial fluid pressure and improvement in chemotherapy delivery formed the rationale for combining chemotherapy with thalidomide in several clinical trials.

In a French phase III randomized controlled trial by Pujol et al. (56), 119 patients with ED-SCLC were treated with 2 cycles of PCDE (cisplatin/cyclophosphamide/ epidoxorubicin/etoposide). Those who achieved an objective response (97 patients) were randomly assigned to receive 4 additional cycles of PCDE plus oral thalidomide $400 \mathrm{mg} / \mathrm{d}$ or placebo. Median treatment duration did not differ between arms (4.5 months for placebo vs. 4.9 months for thalidomide). After a minimum follow up of 3 years, OS was longer with thalidomide than placebo, without achieving statistical significance (11.7 vs. 8.7 months). Of note, patients with PS 1-2 derived a statistically significant survival benefit (HR 0.59; 95\% CI, 0.37-0.92; $\mathrm{P}=0.02)$. Similar results were observed for PFS (6.6 months for thalidomide vs. 6.4 months for placebo, HR 0.74; 95\% CI, 0.49-1.12; $\mathrm{P}=0.15$ ). Thalidomide was more toxic than placebo, as grade $2-4$ neurotoxicity and constipation were three times higher in the thalidomide than in the placebo group and drug discontinuation was more frequent in the experimental arm.

In another phase III randomized controlled trial by Lee et al. (57), 724 patients (51\% LD and 49\% ED-SCLC) were randomized to receive carboplatin and etoposide plus thalidomide $100 \mathrm{mg}$ daily (with the option of escalation up to $200 \mathrm{mg}$ daily if well tolerated) or placebo. Thoracic radiotherapy and PCI were given to patients with objective response. The trial's primary endpoint was OS. After a median follow-up of 37 months, OS was 10.5 months in the placebo group $v s .10 .1$ months in the thalidomide group. PFS and ORR were similar between groups. Although there was no difference in severe toxicity between groups, neurotoxicity, rash, constipation and thrombotic events were more frequent in the thalidomide group.

\section{Other molecules}

Ziv-aflibercept is a human fusion protein composed of high affinity binding domains from the extracellular domain of VEGFR1 and VEGFR2 fused to the Fc fragment of human immunoglobulin G1. Its mechanism of action involves binding to circulating VEGF-A, VEGF-B and placental growth factor. It has been approved for use in metastatic colorectal cancer. In the Southwest Oncology Group (SWOG) S0802 trial (58), 192 patients who had progressed after a platinum based regimen were randomly assigned to receive intravenous topotecan and ziv-aflibercept $6 \mathrm{mg} / \mathrm{kg}$ intravenously once every 21 days or topotecan alone. The trial's primary endpoint was 3-month PFS and it was significantly prolonged for the combination than the topotecan arm ( $26 \%$ vs. $12 \%, \mathrm{P}=0.01)$; median $\mathrm{PFS}$ was 1.6 and 1.3 months respectively. In the subgroup of patients with platinum sensitive relapse, this difference was less 
pronounced and did not reach statistical significance. Median OS was 5.4 months for the combination and 4.4 months for the topotecan alone arm, which was not statistically significant, regardless of platinum sensitivity. There were more grade 3-5 toxicities in the combination arm and the rate of discontinuation was higher as well (18\% vs. 6\%).

Vandetanib is an oral inhibitor of VEGFR2 and to a lesser extent of EGFR. In a trial by Arnold et al. 107 patients who had achieved objective response after a minimum of four cycles of platinum based chemotherapy were randomly assigned to maintenance treatment with vandetanib $300 \mathrm{mg}$ daily or placebo. Two thirds of the study population had received thoracic radiotherapy. Median duration of treatment was 7 weeks for the vandetanib and 12 weeks for the placebo groups. After a median follow up of 13.5 months, the trial's primary endpoint, PFS was similar between arms (2.7 months for vandetanib vs. 2.8 months for placebo, HR 1.01, $\mathrm{P}=0.51$ ). OS was also similar between arms (10.6 vs. 11.9 months), but severe (grade 3-5) toxicity was more frequent in the vandetanib group. Adverse events of interest such as QTc prolongation, hypertension, diarrhea and rash were more frequent in the vandetanib arm (59).

Cediranib is a small molecule inhibitor of VEGF receptors $1-3$. In a phase II trial it was evaluated in 25 patients in the second line setting. The drug was given at the $45 \mathrm{mg}$ daily dose for the first 12 patients who entered the trial, but the dose was amended to $30 \mathrm{mg}$ because of toxicity concerns. For the patients on the high dose level, median number of cycles received was less than one (each cycle consisted of 4 weeks of treatment), whereas the median number of cycles received at the low dose level was 2 . No objective response was noted, but 13 patients $(52 \%)$ experienced disease stabilization. Common toxicity involved nausea, vomiting, fatigue, diarrhea, proteinuria and hypertension (60).

Sorafenib is a multikinase inhibitor of both intracellular Raf kinases as well as VEGF receptors, PDGFR-b, c-kit, Flt-3 and RET. It has been approved for use in renal cell carcinoma, hepatocellular carcinoma (HCC) and well differentiated thyroid cancer. In the phase II SWOG 0435 trial (61), 83 eligible patients with ED-SCLC progressing after platinum-based chemotherapy were treated with sorafenib $400 \mathrm{mg}$ twice daily. ORR was $6 \%$ (11\% for the platinum sensitive and $2 \%$ for the platinum refractory patients). Median PFS was 2 months and median OS was 5.3 and 6.7 months for the platinum-refractory and platinumsensitive patients, respectively.

Pazopanib is a TKI that inhibits VEGFR-1, -2 and -3 , PDGFR, FGFR-1 and -3 and c-kit. It is approved for use in metastatic renal cell carcinoma and advanced soft tissue sarcomas. In a phase II trial conducted by the Hellenic Oncology Research Group presented at the 16th World Conference on Lung Cancer (62), a two cohort design of platinum-sensitive and platinum-resistant disease was used. Patients progressing after one chemotherapy regimen $(25 \%$ had limited stage disease) were treated with pazopanib $800 \mathrm{mg}$ daily. In the platinum-sensitive cohort, of the 39 patients treated, 7 patients (18\%) achieved partial response and 16 patients $(41 \%)$ had stable disease, for a DCR of $59 \%$. Median PFS was 5.5 months and median OS was 8.0 months. Accrual in the platinum-resistant cohort was not completed because of futility after the interim analysis. Of the 19 patients accrued, 1 patient $(5.25 \%)$ achieved partial response and four stable disease. Median PFS was 2 months and median OS was 4 months.

Recently, a two-stage phase II study of nintedanib, an oral multikinase inhibitor, in patients with relapsed SCLC was published (63). Results were disappointing, as ORR was only $5 \%$, PFS 1.0 months and OS 9.8 months and the trial was discontinued before full accrual.

Rovalpituzumab-tesirine is an antibody-drug conjugate (ADC) that binds to the ligand of delta-like protein 3 (DLL3), an atypical Notch ligand that is highly expressed in approximately two-thirds of SCLCs (68). In a phase I trial, 73 patients with recurrent SCLC were treated with rovalpituzumab-tesirine; among 27 DLL3-high positive patients, $44 \%$ had a partial response and $34 \%$ achieved stable disease. Additionally, in the third-line setting, the response rate was $45 \%$ in patients whose tumors had DLL3-high expression (64). Accordingly, a trial with rovalpituzumab-tesirine in the third line setting and later (NCT02674568) has been initiated.

\section{Discussion}

Despite the existing biologic rationale for targeting angiogenesis in SCLC, results of clinical trials have been largely negative. This lack of efficacy and subsequent pessimism is reflected on the number of ongoing trials of such agents: a search at the clinicaltrials.gov website revealed only 3 trials of anti-angiogenesis agents in SCLC that are currently recruiting participants (maintenance pazopanib, NCT01797874; third line or later rovalpituzumab-tesirine, NCT02674568; cediranib combined with olaparib in various tumors including SCLC, NCT02498613) and clinical research interest has now shifted towards the manipulation of tumor-host immune interactions. 
An important consideration when assessing the efficacy of anti-angiogenic strategies is the lack of predictive biomarkers. A subset of patients that derive benefit may exist but the inability to recognize them results in everyone being treated with the same agents: hardly a paradigm of precision medicine. Several biomarkers have been proposed, such as treatment-related hypertension, VEGF levels, circulating endothelial and endothelial progenitor cells levels, tumor endothelial markers and functional imaging, but none has been prospectively shown to select and accurately predict the response to angiogenesis inhibitors (69). Furthermore, despite the fact that several components of the VEGF pathway have been demonstrated to exhibit significant prognostic power in SCLC, none is used for risk stratification purposes in daily practice.

The lack of efficacy of angiogenesis inhibition in SCLC may be caused by the amplification of pro-angiogenic genes, the secretion of multiple pro-angiogenic factors, the promotion of tumor invasiveness and the activation of parallel pathways and mechanisms that bypass the tumor's dependence on neovascularization for its progression. One proposed alternative mechanism, independent of the formation of endothelial vessels, is vasculogenic mimicry (VM) which describes the ability of tumor cells with "stemness" characteristics to independently form vascular networks and to promote invasion and metastatic potential (70). The main stimulus for VM is hypoxia and the process resembles epithelial-to-mesenchymal transition (EMT), since the tumor cells with stem-like plasticity adopt endothelial characteristics (71). Hypoxia promotes an undifferentiated cell state by the activation of a non-canonical crosstalk between HIF- $1 \alpha$ and Notch signaling pathways and can further stimulate VM through the generation of mitochondrial reactive oxygen species (ROS) (72,73). In large-cell lung cancer (LCLC), VM has been associated with elevated expression of urokinase plasminogen activator receptor (uPAR), EMT-regulated Twist and Snail and poor prognosis. Additionally, in HCC, the Twist-mediated downregulation of miR-27a-3p is essential for metastasis and VM (74).

VM has been observed in SCLC, where it is driven by vascular endothelial (VE)-cadherin and it is correlated with poor survival (75). Interestingly, VE-cadherin expression has been demonstrated in SCLC circulating tumor cells as well, therefore indicating that VM may have a role in metastases formation (76). In summary, VM provides an escape mechanism to angiogenesis inhibition and may represent a potentially targetable process.
Cancer stem cells (CSCs) are thought to be major contributors to the aggressive phenotype of SCLC (77). This heterogeneous and diverse pool of tumor cells has been linked with the production of pro-angiogenic signals such as VEGF-A (78). On the other hand, VEGF affects CSCs both by creating a perivascular niche for them and directly through neuropilin-1 in an autocrine loop (79). This interplay between angiogenesis and tumor initiating cells offers the rationale for combining anti-angiogenetic agents with drugs that target CSCs (80). Novel compounds, such as CVM-1118, targeting VM and the associated stem cell and drug resistance phenotypes are under development (81).

Besides targeting VM and CSCs, other novel alternative strategies in an effort to improve the results of antiangiogenesis therapy have been proposed. These include the use of metronomic chemotherapy that targets endothelial cells, the concurrent inhibition of multiple pro-angiogenic molecules, the disruption of tumor vasculature using DLL4 inhibitors, the inhibition of the recruitment of bone marrow derived cells by the tumor, the blockade of neuropilins thus interrupting vessel maturation and the synchronous inhibition of factors that promote tumor progression, such as MET proto-oncogene, receptor tyrosine kinase (MET) $(82,83)$. Importantly, simultaneous blockade of multiple mechanisms is crucial in order to effectively suppress redundant escape processes and to prevent the emergence of resistance and disease progression.

\section{Conclusions}

In conclusion, results from a number of relatively small nonrandomized and a few randomized clinical trials indicate that contemporary strategies of angiogenesis inhibition are ineffective in controlling SCLC. The recognition of multiple escape mechanisms and the development and clinical evaluation of novel treatments may restore interest in pursuing these agents in this highly aggressive and lethal disease. These efforts should include predictive biomarkers in order to optimize clinical outcomes.

\section{Acknowledgements}

None.

\section{Footnote}

Conflicts of Interest: The authors have no conflicts of interest to declare. 


\section{References}

1. Govindan R, Page N, Morgensztern D, et al. Changing epidemiology of small-cell lung cancer in the United States over the last 30 years: analysis of the surveillance, epidemiologic, and end results database. J Clin Oncol 2006;24:4539-44.

2. Fox W, Scadding JG. Medical Research Council comparative trial of surgery and radiotherapy for primary treatment of small-celled or oat-celled carcinoma of bronchus. Ten-year follow-up. Lancet 1973;2:63-5.

3. Lassen U, Osterlind K, Hansen M, et al. Longterm survival in small-cell lung cancer: posttreatment characteristics in patients surviving 5 to $18+$ years-an analysis of 1,714 consecutive patients. J Clin Oncol 1995;13:1215-20.

4. Lara PN Jr, Natale R, Crowley J, et al. Phase III trial of irinotecan/cisplatin compared with etoposide/cisplatin in extensive-stage small-cell lung cancer: clinical and pharmacogenomic results from SWOG S0124. J Clin Oncol 2009;27:2530-5.

5. Roth BJ, Johnson DH, Einhorn LH, et al. Randomized study of cyclophosphamide, doxorubicin, and vincristine versus etoposide and cisplatin versus alternation of these two regimens in extensive small-cell lung cancer: a phase III trial of the Southeastern Cancer Study Group. J Clin Oncol 1992;10:282-91.

6. Ihde DC, Mulshine JL, Kramer BS, et al. Prospective randomized comparison of high-dose and standarddose etoposide and cisplatin chemotherapy in patients with extensive-stage small-cell lung cancer. J Clin Oncol 1994;12:2022-34.

7. Ardizzoni A, Tjan-Heijnen VC, Postmus PE, et al. Standard versus intensified chemotherapy with granulocyte colony-stimulating factor support in small-cell lung cancer: a prospective European Organization for Research and Treatment of Cancer-Lung Cancer Group Phase III Trial-08923. J Clin Oncol 2002;20:3947-55.

8. Thatcher N, Girling DJ, Hopwood P, et al. Improving survival without reducing quality of life in small-cell lung cancer patients by increasing the dose-intensity of chemotherapy with granulocyte colony-stimulating factor support: results of a British Medical Research Council Multicenter Randomized Trial. Medical Research Council Lung Cancer Working Party. J Clin Oncol 2000;18:395-404.

9. Bozcuk H, Artac M, Ozdogan M, et al. Does maintenance/ consolidation chemotherapy have a role in the management of small cell lung cancer (SCLC)? A metaanalysis of the published controlled trials. Cancer 2005;104:2650-7.

10. Niell HB, Herndon JE 2nd, Miller AA, et al. Randomized phase III intergroup trial of etoposide and cisplatin with or without paclitaxel and granulocyte colony-stimulating factor in patients with extensive-stage small-cell lung cancer: Cancer and Leukemia Group B Trial 9732. J Clin Oncol 2005;23:3752-9.

11. Pujol JL, Daurès JP, Rivière A, et al. Etoposide plus cisplatin with or without the combination of 4 '-epidoxorubicin plus cyclophosphamide in treatment of extensive small-cell lung cancer: a French Federation of Cancer Institutes multicenter phase III randomized study. J Natl Cancer Inst 2001;93:300-8.

12. O'Brien ME, Ciuleanu TE, Tsekov H, et al. Phase III trial comparing supportive care alone with supportive care with oral topotecan in patients with relapsed small-cell lung cancer. J Clin Oncol 2006;24:5441-7.

13. von Pawel J, Schiller JH, Shepherd FA, et al. Topotecan versus cyclophosphamide, doxorubicin, and vincristine for the treatment of recurrent small-cell lung cancer. J Clin Oncol 1999;17:658-67.

14. von Pawel J, Jotte R, Spigel DR, et al. Randomized phase III trial of amrubicin versus topotecan as second-line treatment for patients with small-cell lung cancer. J Clin Oncol 2014;32:4012-9.

15. Hanahan D, Weinberg RA. Hallmarks of cancer: the next generation. Cell 2011;144:646-74.

16. Nagy JA, Chang SH, Shih SC, et al. Heterogeneity of the tumor vasculature. Semin Thromb Hemost 2010;36:321-31.

17. Baeriswyl V, Christofori G. The angiogenic switch in carcinogenesis. Semin Cancer Biol 2009;19:329-37.

18. Ferrara N. Vascular endothelial growth factor. Arterioscler Thromb Vasc Biol 2009;29:789-91.

19. Lobov IB, Renard RA, Papadopoulos N, et al. Deltalike ligand 4 (Dll4) is induced by VEGF as a negative regulator of angiogenic sprouting. Proc Natl Acad Sci U S A 2007;104:3219-24.

20. Qian BZ, Pollard JW. Macrophage diversity enhances tumor progression and metastasis. Cell 2010;141:39-51.

21. Ellis LM, Reardon DA. Cancer: The nuances of therapy. Nature 2009;458:290-2.

22. Bishop-Bailey D. Tumour vascularisation: a druggable target. Curr Opin Pharmacol 2009;9:96-101.

23. Schneider BJ, Kalemkerian GP. Personalized Therapy of Small Cell Lung Cancer. Adv Exp Med Biol 2016;890:149-74. 
24. Lund EL, Thorsen C, Pedersen MW, et al. Relationship between vessel density and expression of vascular endothelial growth factor and basic fibroblast growth factor in small cell lung cancer in vivo and in vitro. Clin Cancer Res 2000;6:4287-91.

25. Lucchi M, Mussi A, Fontanini G, et al. Small cell lung carcinoma (SCLC): the angiogenic phenomenon. Eur J Cardiothorac Surg 2002;21:1105-10.

26. Wan J, Chai H, Yu Z, et al. HIF-1 $\alpha$ effects on angiogenic potential in human small cell lung carcinoma. J Exp Clin Cancer Res 2011;30:77.

27. Litz J, Krystal GW. Imatinib inhibits c-Kit-induced hypoxia-inducible factor-1alpha activity and vascular endothelial growth factor expression in small cell lung cancer cells. Mol Cancer Ther 2006;5:1415-22.

28. Schneider BJ, Kalemkerian GP, Ramnath N, et al. Phase II trial of imatinib maintenance therapy after irinotecan and cisplatin in patients with c-Kit-positive, extensive-stage small-cell lung cancer. Clin Lung Cancer 2010;11:223-7.

29. Nowak K, Rafat N, Belle S, et al. Circulating endothelial progenitor cells are increased in human lung cancer and correlate with stage of disease. Eur J Cardiothorac Surg 2010;37:758-63.

30. Lin Q, Xue L, Tian T, et al. Prognostic value of serum IL17 and VEGF levels in small cell lung cancer. Int J Biol Markers 2015;30:e359-63.

31. Salven P, Ruotsalainen T, Mattson K, et al. High pretreatment serum level of vascular endothelial growth factor (VEGF) is associated with poor outcome in small-cell lung cancer. Int J Cancer 1998;79:144-6.

32. Zhan P, Wang J, Lv XJ, et al. Prognostic value of vascular endothelial growth factor expression in patients with lung cancer: a systematic review with meta-analysis. J Thorac Oncol 2009;4:1094-103.

33. Tanno S, Ohsaki Y, Nakanishi K, et al. Human small cell lung cancer cells express functional VEGF receptors, VEGFR-2 and VEGFR-3.Lung Cancer 2004;46:11-9.

34. Zhang CL, Ge SL, Yang N, et al. Elevated Serum Level of Angiopoietin-2 as a Potential Marker for Poor Prognosis in Small Cell Lung Cancer. Tohoku J Exp Med 2015;236:305-9.

35. Cañadas I, Taus Á, Villanueva X, et al. Angiopoietin-2 is a negative prognostic marker in small cell lung cancer. Lung Cancer 2015;90:302-6.

36. Hong DS, Kurzrock R, Mulay M, et al. A phase 1b, open-label study of trebananib plus bevacizumab or motesanib in patients with solid tumours. Oncotarget 2014;5:11154-67.
37. Ruotsalainen T, Joensuu H, Mattson K, et al. High pretreatment serum concentration of basic fibroblast growth factor is a predictor of poor prognosis in small cell lung cancer. Cancer Epidemiol Biomarkers Prev 2002;11:1492-5.

38. Ueno K, Inoue $Y$, Kawaguchi T, et al. Increased serum levels of basic fibroblast growth factor in lung cancer patients: relevance to response of therapy and prognosis. Lung Cancer 2001;31:213-9.

39. $\mathrm{Hu} \mathrm{M}, \mathrm{Hu} \mathrm{Y}, \mathrm{He} \mathrm{J}$, et al. Prognostic Value of Basic Fibroblast Growth Factor (bFGF) in Lung Cancer: A Systematic Review with Meta-Analysis. PLoS One 2016;11:e0147374.

40. Zhang L, Yu H, Badzio A, et al. Fibroblast Growth Factor Receptor 1 and Related Ligands in Small-Cell Lung Cancer. J Thorac Oncol 2015;10:1083-90.

41. Schultheis AM, Bos M, Schmitz K, et al. Fibroblast growth factor receptor 1 (FGFR1) amplification is a potential therapeutic target in small-cell lung cancer. Mod Pathol 2014;27:214-21.

42. Pardo OE, Latigo J, Jeffery RE, et al. The fibroblast growth factor receptor inhibitor PD173074 blocks small cell lung cancer growth in vitro and in vivo. Cancer Res 2009;69:8645-51.

43. Ready NE, Dudek AZ, Pang HH, et al. Cisplatin, irinotecan, and bevacizumab for untreated extensive-stage small-cell lung cancer: CALGB 30306, a phase II study. J Clin Oncol 2011;29:4436-41.

44. Spigel DR, Townley PM, Waterhouse DM, et al. Randomized phase II study of bevacizumab in combination with chemotherapy in previously untreated extensive-stage small-cell lung cancer: results from the SALUTE trial. J Clin Oncol 2011;29:2215-22.

45. Horn L, Dahlberg SE, Sandler AB, et al. Phase II study of cisplatin plus etoposide and bevacizumab for previously untreated, extensive-stage small-cell lung cancer: Eastern Cooperative Oncology Group Study E3501. J Clin Oncol 2009;27:6006-11.

46. Spigel DR, Greco FA, Zubkus JD, et al. Phase II trial of irinotecan, carboplatin, and bevacizumab in the treatment of patients with extensive-stage small-cell lung cancer. J Thorac Oncol 2009;4:1555-60.

47. Pujol JL, Lavole A, Quoix E, et al. Randomized phase II-III study of bevacizumab in combination with chemotherapy in previously untreated extensive small-cell lung cancer: results from the IFCT-0802 trial†. Ann Oncol 2015;26:908-14.

48. Petrioli R, Roviello G, Laera L, et al. Cisplatin, Etoposide, 
and Bevacizumab Regimen Followed by Oral Etoposide and Bevacizumab Maintenance Treatment in Patients With Extensive-Stage Small Cell Lung Cancer: A SingleInstitution Experience. Clin Lung Cancer 2015;16:e229-34.

49. Jalal S, Bedano P, Einhorn L, et al. Paclitaxel plus bevacizumab in patients with chemosensitive relapsed small cell lung cancer: a safety, feasibility, and efficacy study from the Hoosier Oncology Group. J Thorac Oncol 2010;5:2008-11.

50. Mountzios G, Emmanouilidis C, Vardakis N, et al. Paclitaxel plus bevacizumab in patients with chemoresistant relapsed small cell lung cancer as salvage treatment: a phase II multicenter study of the Hellenic Oncology Research Group. Lung Cancer 2012;77:146-50.

51. Spigel DR, Waterhouse DM, Lane S, et al. Efficacy and safety of oral topotecan and bevacizumab combination as second-line treatment for relapsed small-cell lung cancer: an open-label multicenter single-arm phase II study. Clin Lung Cancer 2013;14:356-63.

52. Schneider BJ, Gadgeel SM, Ramnath N, et al. Phase II trial of sunitinib maintenance therapy after platinum-based chemotherapy in patients with extensive-stage small cell lung cancer. J Thorac Oncol 2011;6:1117-20.

53. Spigel DR, Greco FA, Rubin MS, et al. Phase II study of maintenance sunitinib following irinotecan and carboplatin as first-line treatment for patients with extensive-stage small-cell lung cancer. Lung Cancer 2012;77:359-64.

54. Ready NE, Pang HH, Gu L, et al. Chemotherapy With or Without Maintenance Sunitinib for Untreated ExtensiveStage Small-Cell Lung Cancer: A Randomized, DoubleBlind, Placebo-Controlled Phase II Study-CALGB 30504 (Alliance). J Clin Oncol 2015;33:1660-5.

55. Han JY, Kim HY, Lim KY, et al. A phase II study of sunitinib in patients with relapsed or refractory small cell lung cancer. Lung Cancer 2013;79:137-42.

56. Pujol JL, Breton JL, Gervais R, et al. Phase III doubleblind, placebo-controlled study of thalidomide in extensive-disease small-cell lung cancer after response to chemotherapy: an intergroup study FNCLCC cleo04 IFCT 00-01. J Clin Oncol 2007;25:3945-51.

57. Lee SM, Woll PJ, Rudd R, et al. Anti-angiogenic therapy using thalidomide combined with chemotherapy in small cell lung cancer: a randomized, double-blind, placebocontrolled trial. J Natl Cancer Inst 2009;101:1049-57.

58. Allen JW, Moon J, Redman M, et al. Southwest Oncology Group S0802: a randomized, phase II trial of weekly topotecan with and without ziv-aflibercept in patients with platinum-treated small-cell lung cancer. J Clin Oncol
2014;32:2463-70.

59. Arnold AM, Seymour L, Smylie M, et al. Phase II study of vandetanib or placebo in small-cell lung cancer patients after complete or partial response to induction chemotherapy with or without radiation therapy: National Cancer Institute of Canada Clinical Trials Group Study BR.20. J Clin Oncol 2007;25:4278-84.

60. Ramalingam SS, Belani CP, Mack PC, et al. Phase II study of Cediranib (AZD 2171), an inhibitor of the vascular endothelial growth factor receptor, for second-line therapy of small cell lung cancer (National Cancer Institute \#7097). J Thorac Oncol 2010;5:1279-84.

61. Gitlitz BJ, Moon J, Glisson BS, et al. Sorafenib in platinum-treated patients with extensive stage small cell lung cancer: a Southwest Oncology Group (SWOG 0435) phase II trial. J Thorac Oncol 2010;5:1835-40.

62. Kotsakis A, Karavasilis V, Agelaki S, et al. Pazopanib as Second Line Treatment of Platinum Sensitive SCLC Patients: A Multicenter Phase II Trial of the Hellenic Oncology Research Group. 16th World Conference on Lung Cancer. Denver, Colorado, USA. 2015 Sep 6-9.

63. Han JY, Kim HY, Lim KY, et al. A phase II study of nintedanib in patients with relapsed small cell lung cancer. Lung Cancer 2016;96:108-12.

64. Pietanza MC. Safety, activity, and response durability assessment of single agent rovalpituzumab tesirine, a deltalike protein 3 (DLL3)-targeted antibody drug conjugate (ADC), in small cell lung cancer. European Cancer Congress 2015. Vienna, Austri. 2015 Sep 25-29.

65. Rini BI, Michaelson MD, Rosenberg JE, et al. Antitumor activity and biomarker analysis of sunitinib in patients with bevacizumab-refractory metastatic renal cell carcinoma. J Clin Oncol 2008;26:3743-8.

66. Prior JO, Montemurro M, Orcurto MV, et al. Early prediction of response to sunitinib after imatinib failure by $18 \mathrm{~F}$-fluorodeoxyglucose positron emission tomography in patients with gastrointestinal stromal tumor. J Clin Oncol 2009;27:439-45.

67. Jin E, Fujiwara M, Pan X, et al. Protease-activated receptor (PAR)-1 and PAR-2 participate in the cell growth of alveolar capillary endothelium in primary lung adenocarcinomas. Cancer 2003;97:703-13.

68. Saunders LR, Bankovich AJ, Anderson WC, et al. A DLL3-targeted antibody-drug conjugate eradicates highgrade pulmonary neuroendocrine tumor-initiating cells in vivo. Sci Transl Med 2015;7:302ra136.

69. Pircher A, Jöhrer K, Kocher F, et al. Biomarkers of evasive resistance predict disease progression in cancer 
patients treated with antiangiogenic therapies. Oncotarget 2016;7:20109-23.

70. Li S, Meng W, Guan Z, et al. The hypoxia-related signaling pathways of vasculogenic mimicry in tumor treatment. Biomed Pharmacother 2016;80:127-35.

71. Sun B, Zhang D, Zhao N, et al. Epithelial-to-endothelial transition and cancer stem cells: two cornerstones of vasculogenic mimicry in malignant tumors. Oncotarget 2016. [Epub ahead of print].

72. Borggrefe T, Lauth M, Zwijsen A, et al. The Notch intracellular domain integrates signals from $\mathrm{Wnt}$, Hedgehog, TGF $\beta / B M P$ and hypoxia pathways. Biochim Biophys Acta 2016;1863:303-13.

73. Comito G, Calvani M, Giannoni E, et al. HIF-1 $\alpha$ stabilization by mitochondrial ROS promotes Metdependent invasive growth and vasculogenic mimicry in melanoma cells. Free Radic Biol Med 2011;51:893-904.

74. Li Y, Sun B, Zhao X, et al. Subpopulations of uPAR+ contribute to vasculogenic mimicry and metastasis in large cell lung cancer. Exp Mol Pathol 2015;98:136-44.

75. Trapani F, Metcalf R, Hodgkinson C, et al. Vasculogenic mimicry in small-cell lung cancer. NCRI Cancer Conference. Liverpool, UK, 2015 Nov 1-4.

76. Trapani F, Williamson S, Metcalf R, et al. Genetic,

Cite this article as: Stratigos M, Matikas A, Voutsina A, Mavroudis D, Georgoulias V. Targeting angiogenesis in small cell lung cancer. Transl Lung Cancer Res 2016;5(4):389-400. doi: 10.21037/tlcr.2016.08.04 phenotypic and functional characterisation of vasculogenic mimicry in small-cell lung cancer. AACR Annual Meeting. New Orleans, USA, 2016 Apr 16-20.

77. Codony-Servat J, Verlicchi A, Rosell R. Cancer stem cells in small cell lung cancer. Transl Lung Cancer Res 2016;5:16-25.

78. Fantozzi A, Gruber DC, Pisarsky L, et al. VEGF-mediated angiogenesis links EMT-induced cancer stemness to tumor initiation. Cancer Res 2014;74:1566-75.

79. Beck B, Driessens G, Goossens S, et al. A vascular niche and a VEGF-Nrp1 loop regulate the initiation and stemness of skin tumours. Nature 2011;478:399-403.

80. Zhao Y, Bao Q, Renner A, et al. Cancer stem cells and angiogenesis. Int J Dev Biol 2011;55:477-82.

81. Hendrix MJ, Seftor EA, Seftor RE, et al. Tumor cell vascular mimicry: Novel targeting opportunity in melanoma. Pharmacol Ther 2016;159:83-92.

82. Sennino B, McDonald DM. Controlling escape from angiogenesis inhibitors. Nat Rev Cancer 2012;12:699-709.

83. Loges S, Schmidt T, Carmeliet P. Mechanisms of resistance to anti-angiogenic therapy and development of third-generation anti-angiogenic drug candidates. Genes Cancer 2010;1:12-25. 